\title{
Undivided Retromandibular Vein Leading to the Absence of External Jugular Vein
}

\author{
Y. Lakshmisha Rao ${ }^{1}$ Vaishnavi Ballal ${ }^{2} \quad$ B. V. Murlimanju ${ }^{1} \quad$ Mangala M. Pai $^{1}$ Mamatha Tonse $^{1}$ \\ Ashwin Krishnamurthy ${ }^{3}$
}

${ }^{1}$ Department of Anatomy, Kasturba Medical College, Mangalore, A Constituent Institution of Manipal Academy of Higher Education, Manipal, Karnataka, India

${ }^{2}$ Kasturba Medical College, Mangalore, A Constituent Institution of Manipal Academy of Higher Education, Manipal, Karnataka, India

${ }^{3}$ Department of Anatomy, American University of Antigua, College of Medicine, Antigua, West Indies

\author{
Address for correspondence B. V. Murlimanju, MD, Department of \\ Anatomy, Kasturba Medical College, A constituent unit of MAHE, \\ Bejai, Mangalore-575004, Karnataka State, India \\ (e-mail: flutesnowmm@gmail.com).
}

J Morphol Sci 2018;35:225-228.

\begin{abstract}
Keywords

- common trunk

- external jugular vein

- retromandibular vein

We report a case of undivided retromandibular vein (RV) that was associated with the absence of external jugular vein (EJV). This variation in the jugular venous system was observed on the right side of the neck of a male cadaver. The posterior auricular vein drained into the RV, which joined the facial vein resulting in a common trunk. This common trunk then drained directly into the internal jugular vein (IJV) thus bypassing the whole venous drainage area of the EJV. It is believed that the knowledge about the venous variations, as in the present case, is enlightening to the clinicians. The preoperative evaluation of the venous system is essential to prevent complications like catastrophic bleeding and embolism. The knowledge may guide the emergency physician for a quicker cannulation of the veins.
\end{abstract}

\section{Introduction}

The venous system of the embryo is mainly formed by the cardinal veins during the early stages of the intrauterine life. The left and right anterior cardinal veins drain the cephalic part and the left and right posterior cardinal veins drain the caudal part of the embryo. ${ }^{1}$ The anterior cardinal veins afford the primary venous drainage of head and eventually forms the internal jugular vein (IJV), while the plexus of veins in the face form the external jugular vein (EJV). The jugular veins are the major components of the head and neck venous system. The retromandibular vein (RV) is formed within the parotid gland by the union of superficial temporal and maxillary veins. ${ }^{2}$ The RV later divides into anterior and posterior divisions. Its anterior division receives the facial vein to form common facial vein, which eventually opens into IJV. The union of its posterior division with the posterior auricular vein forms the EJV, which drains into the subclavian vein. ${ }^{2}$

received

September 13, 2017

accepted

September 10, 2018

published online

November 8, 2018
DOI https://doi.org/ 10.1055/s-0038-1675760. ISSN 2177-0298.
The course and structure of the veins are more variable than those of the arteries. ${ }^{3}$ The knowledge of such variations is extremely essential for the clinical practice. The major veins of the neck are often used as the standard anatomical landmarks during surgical procedures. However, variations in the anatomy of these veins may have significant clinical outcomes. The IJV is used to measure the jugular venous pressure (JVP) during the routine physical examination. The EJV is the preferred route for cannulation to administer intravenous fluids, drugs and blood products. The EJV is also used to collect blood samples, ${ }^{4}$ and also has been used as a recipient for free flap microsurgeries and venous manometers. ${ }^{5}$ The EJV can be made more dilated and clearly visible during clinical examination by the using the Valsava maneuver. ${ }^{6}$ Since the EJV has various clinical implications, its absence may cause difficulties during routine general physical examination, diagnostic, and therapeutic procedures. In this article, we report a case of

Copyright (c) 2018 by Thieme Revinter Publicações Ltda, Rio de Janeiro, Brazil

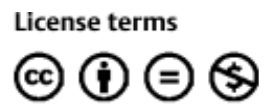


undivided RV, which led to the absence of EJV over the right neck.

\section{Case Report}

During the routine dissection of a male cadaver, aged 72 years, a variation in the venous system over the right side of the face and neck was observed. The right RV was undivided; however, its formation within the parotid gland was normal. The posterior auricular vein drained into the RV (-Fig. 1) to form a common trunk (CT1), just above the angle of the mandible. Near the angle of the mandible, the CT1 joined the facial vein to form another common trunk (CT2; - Fig. 1). The CT2 drained directly into the IJV. The IJV joined the subclavian vein, thus bypassing the whole venous drainage area of the EJV. The variant pattern in the right jugular venous system observed in the present case is schematically represented in -Fig. 2. The arterial pattern of the right side of the head and neck was found normal in this cadaver. The left side of the neck showed the usual morphological pattern of arteries and veins.

\section{Discussion}

There are few anatomical studies available in the scientific literature about the EJV system. Olabu et $\mathrm{al}^{7}$ reported that the EJV was absent in $14.2 \%$ of the studied cases. They observed that variations of the EJV were common on the right side and the male population. It is worth noticing that this present study is also of a male, and on his right side, supporting the

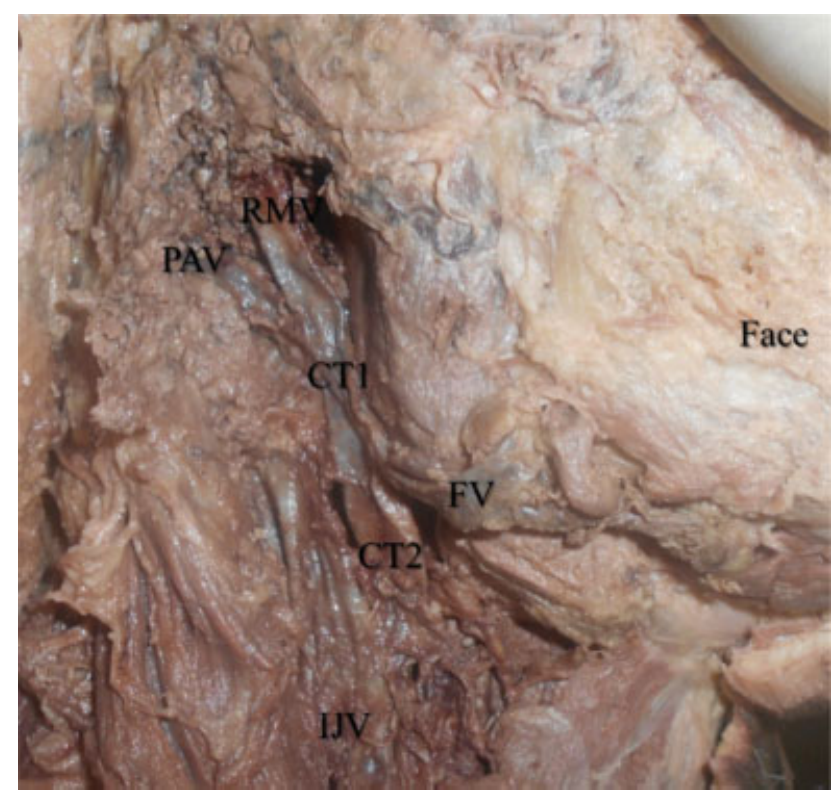

Fig. 1 Right side face and neck of the dissecting room cadaver showing the absence of external jugular vein. The retromandibular vein was undivided, which was receiving the posterior auricular vein to form a common trunk (CT1). The common trunk (CT1) joined the facial vein to form another common trunk (CT2), which drained directly into the internal jugular vein. Abbreviations: CT 1, first common trunk; CT 2, second common trunk; FV, facial vein; IJV, internal jugular vein; PAV, posterior auricular vein; RMV, retromandibular vein.

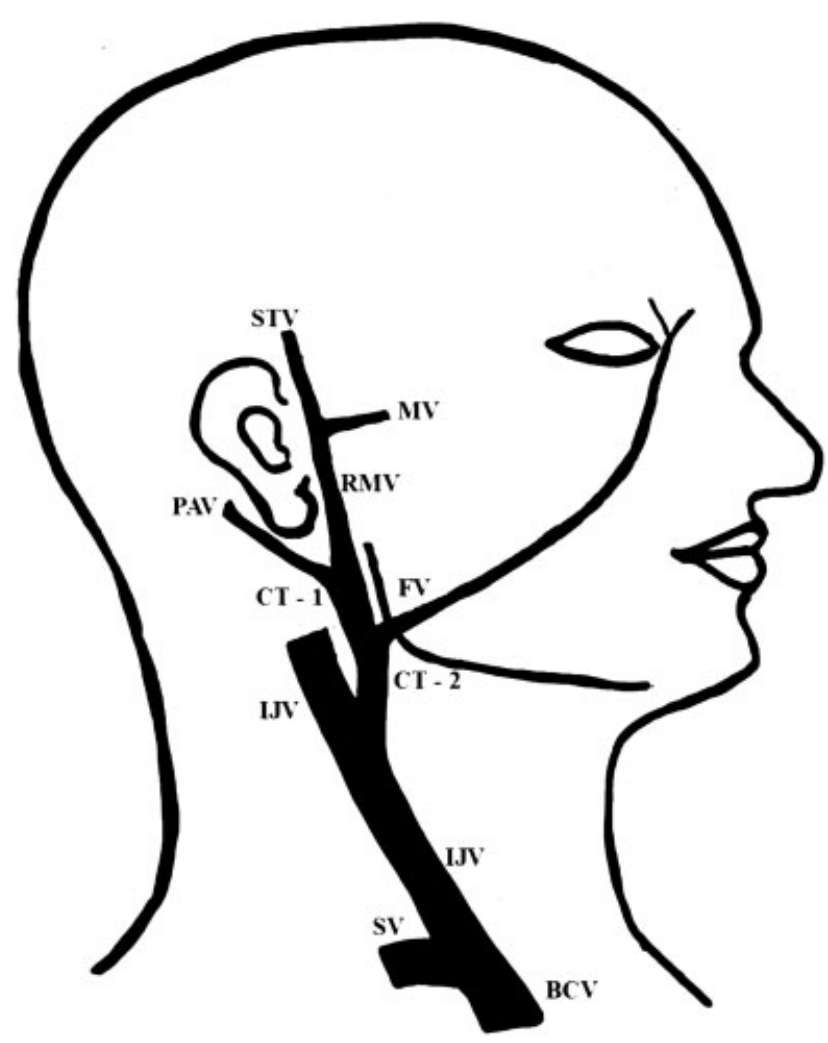

Fig. 2 Schematic representation of the variant pattern in the right jugular venous system, which was observed in the present case. Abbreviations: $\mathrm{BCV}$, brachiocephalic vein; CT 1, first common trunk; CT 2, second common trunk; FV, facial vein; IJV, internal jugular vein; MV, maxillary vein; PAV, posterior auricular vein; RMV, retromandibular vein; STV, superficial temporal vein; SV, splenic vein.

observations of Olabu et al. $^{7}$ Patil et $\mathrm{l}^{8}$ reported an interesting case in which the maxillary vein was split into anterior and posterior divisions. The posterior division joined the superficial temporal vein to form the EJV. Shankar et $\mathrm{al}^{9}$ reported the trifurcation of the RV near the angle of the mandible. Shankar et al ${ }^{9}$ described that these anomalous venous patterns are due to the regression and retention of the venous anastomotic channels during the embryo development.

In the head and neck region, the EJV system drains the scalp and the face. Though the tributaries of the EJV drain a much smaller area in comparison to the IJV, the EJV is an important peripheral vein in the neck to get intravenous access during emergency procedures. The EJV is clinically used as an alternative to the cephalic vein for performing cannulation. ${ }^{10,11}$ It has been described that the risk of air embolism after the central venous cannulation is very minimal with the EJV. Due to the clinical implications of the EJV, its absence prevents the clinician from using this important route to the intravenous access. ${ }^{12}$ It was stated that the variant anatomy of the EJV may not favor the blind surgical procedures of the neck. It is recommended that, if the EJV is not observed during the emergency procedure, the surgeon should not waste time searching for it $^{7}$ and should use another vein because the EJV may be absent on few occasions.

The free flaps form an important modality for plastic and reconstructive surgeries after the excision of the malignancy. 
Veins like the EJV are usually preferred as recipient veins during the oral cavity reconstruction. Preoperative knowledge of the absence of the vein, through imaging, will help the surgeon to plan the procedures with minimal time. ${ }^{5,13}$

The RV acts as a guide while exposing the branches of facial nerve during parotid surgeries and open reduction of temporo-mandibular joint fractures. The RV and its tributaries should be ligated during the surgery to prevent excessive bleeding. ${ }^{3}$ The RV can also be used as a reliable landmark during investigative procedures, like computed tomogram, ultrasonography, and magnetic resonance imaging. ${ }^{14}$ In the present case, the RV was undivided and the knowledge about this type of venous variation is enlightening to the clinician to avoid unnecessary complications. The knowledge is also helpful to cranio-maxillo-facial and head neck surgeons.

Also, to know the course of the EJV is essential during surgical procedures, like portosystemic shunts and transjugular liver biopsy. ${ }^{15}$ It is advised that the surgeons take precautionary measures while ligating the variant veins during the dissection of en-bloc metastatic lymph nodes in the neck. In order to find the perfect choice for the patient, the surgeon should know as many veins as possible. This is because the percutaneous insertion of venous catheters is performed using the anatomic landmarks. The successful placement of these catheters depends on the correct position of the vein. ${ }^{16}$

Near the common carotid artery bifurcation, the common facial vein opens into the IJV. This is an important landmark which helps the surgeons while they explore the carotid sheath. In the present case, the RV was undivided and joined by the posterior auricular vein forming a common trunk. The facial vein opened to the common trunk to form another common trunk, which drained into the IJV. Also, the termination of the vein into the IJV was at a higher level than the bifurcation of the common carotid artery. This variation should be considered during surgical procedures to avoid complications like catastrophic bleeding and air embolism.

The EJV, when not collapsed or thrombosed, becomes a choice if the patient requires an emergency venous access when other veins are collapsed. ${ }^{17}$ Sectioning the EJV is used in parenteral nutrition and chemotherapy administration, and the absence of the EJV should be considered while performing these procedures. ${ }^{18,19}$

Nagase et al ${ }^{20}$ advise that a preoperative evaluation of the venous network patterns of the head and neck region is required before surgery. This can be done by the precise clinical appraisal, followed by color Doppler studies to prevent intraoperative complications.

\section{Conclusion}

A rare case of variation in the jugular venous system is reported. The significance of the present case is a variation in the course of the RV along with the absence of EJV. The undivided RV joined the posterior auricular vein forming a common trunk, which eventually opened into the IJV after joining with the facial vein. These variant trunks have drained the EJV area, since it was not formed. We believe that this report is enlightening to head and neck surgeons, anesthesiologists and cardiovascular surgeons. This has implications in emergency medicine and plastic surgery as well.

Note

Murlimanju B. V. presently works as Associate Professor of Anatomy and is interested in the field of human morphology. He has 35 research articles published on morphology, mainly on the morphology of the human knee joint.

\section{Conflicts of Interest}

None.

\section{References}

1 Singh I. Human Embryology. 10th ed. New Delhi: Jaypee Brothers Medical Publishers; 2014:268-269

2 Williams PL, Ed. Gray's Anatomy, 38th ed. New York: Churchill Livingstone; 1995:1576-1580

3 Choudhary S, Sharma AK, Singh H. Undivided RV continuing as external jugular vein with facial vein draining into it: An anatomical variation. JK Science 2010;12(04):203-204

4 Scales K. Intravenous therapy: a guide to good practice. Br J Nurs 2008;17(19):S4-S12

5 Chauhan NK, Rani A, Chopra J, Rani A, Srivastava AK, Kumar V. Anomalous formation of external jugular vein and its clinical implication. Natl J Maxillofac Surg 2011;2(01):51-53

6 Vinayak AG, Levitt J, Gehlbach B, Pohlman AS, Hall JB, Kress JP. Usefulness of the external jugular vein examination in detecting abnormal central venous pressure in critically ill patients. Arch Intern Med 2006;166(19):2132-2137

7 Olabu BO, Loyal PK, Matiko BW, Nderitu JM, Misiani MK, Ogeng'o JA. Variant anatomy of the external jugular vein. Anatomy Journal of Africa 2015;4(01):518-527

8 Patil J, Kumar N, Swamy RS, D'Souza MR, Guru A, Nayak SB. Absence of retromandibular vein associated with atypical formation of external jugular vein in the parotid region. Anat Cell Biol 2014;47(02):135-137

9 Shankar VV, Chandrashekar D, Chowdapurkar S. Bilateral anatomical variations in the formation, communication and the termination of the RV. J Clin Diagn Res 2012;6(07):1286-1288

10 Di Carlo I, Barbagallo F, Toro A, Sofia M, Lombardo R, Cordio S. External jugular vein cutdown approach, as a useful alternative, supports the choice of the cephalic vein for totally implantable access device placement. Ann Surg Oncol 2005;12(07): 570-573

11 Moini M, Rasouli MR, Kenari MM, Mahmoodi HR. Non-cuffed dual lumen catheters in the external jugular veins versus other central veins for hemodialysis patients. Saudi J Kidney Dis Transpl 2009; 20(01):44-48

12 Cvetko E. A case of left-sided absence and right-sided fenestration of the external jugular vein and a review of the literature. Surg Radiol Anat 2015;37(07):883-886

13 Shenoy V, Saraswathi P, Raghunath G, Karthik JS. Double external jugular vein and other rare venous variations of the head and neck. Singapore Med J 2012;53(12):e251-e253

14 Piagkou M, Tzika M, Paraskevas G, Natsis K. Anatomic variability in the relation between the retromandibular vein and the facial nerve: a case report, literature review and classification. Folia Morphol (Warsz) 2013;72(04):371-375 
15 Gupta N, Jain P, Anshu A, Samta. An undivided retro-mandibular vein receiving facial and posterior auricular vein: case report and clinical repercussion. Indian J Basic Appl Med Res 2013;7(02):710-715

16 Gupta V, Tuli A, Choudhry R, Agarwal S, Mangal A. Facial vein draining into external jugular vein in humans: its variations, phylogenetic retention and clinical relevance. Surg Radiol Anat 2003;25(01):36-41

17 Arquez HF. Unusual venous drainage of the head and neck. Int J Pharma Bio Sci 2014;5(04):965-970
18 Selvi GP, Kumar BS. Variations in the venous drainage pattern of face and neck. Int J Pharma Bio Sci 2013;4(04):150-154

19 Vaida MA, Niculescu V, Motoc A, Bolintineanu S, Sargan I, Niculescu MC. Correlations between anomalies of jugular veins and areas of vascular drainage of head and neck. Rom J Morphol Embryol 2006;47(03):287-290

20 Nagase T, Kobayashi S, Sekiya S, Ohmori K. Anatomic evaluation of the facial artery and vein using color Doppler ultrasonography. Ann Plast Surg 1997;39(01):64-67 